\title{
Prevalence of Metabolic Syndrome and Its Prediction by Simple Adiposity Indices in Thai Adults
}

\author{
Hung Nguyen Ngoc, M.Sc. ${ }^{1}$, Wantanee Kriengsinyos, R.D., Ph.D. ${ }^{1}$, \\ Nipa Rojroongwasinkul, Ph.D. ${ }^{1}$, Wichai Aekplakorn, M.D., Ph.D. ${ }^{2}$ \\ ${ }^{1}$ Institute of Nutrition, Mahidol University, Phutthamonthon, Nakhon Pathom 73170, Thailand. \\ ${ }^{2}$ Faculty of Medicine Ramathibodi Hospital, Mahidol University, Ratchathewi, Bangkok 10400, Thailand. \\ Received 11 August 2020 • Revised 28 September 2020 • Accepted 9 October 2020 • Published online 25 March 2021
}

\begin{abstract}
:
Objective: Thai adults, have increased risk of being diagnosed with metabolic syndrome (MetS). Hence, early discrimination of MetS, with a simple and high accuracy index, appears necessary. However, the application of the discriminating ability of Lipid Accumulation Product (LAP), which is an emergent indicator of central lipid accumulation, to MetS among Thai people has not been investigated. This present study's purposes were to investigate the nationwide prevalence of MetS, and the ability of LAP in discriminating this disorder.
\end{abstract}

Material and Methods: Cross-sectional secondary data analysis was performed in 2018, using primary data from the Thai National Health Examination Survey, 2009. A total of 18,642 Thailanders $\geq 18$ years were recruited. MetS was diagnosed by the National Cholesterol Education Program/Adult Treatment Panel III (NCEP/ATP) and International Diabetes Federation (IDF).

Results: Overall, the prevalence of MetS-NCEP/ATP and MetS-IDF in Thai adults was $20.0 \%$ and $27.0 \%$, respectively. LAP showed outstanding discriminating ability for MetS in both definitions (the cut-off point of 34.38 and $37.96 \mathrm{~cm} . \mathrm{mmol} /$; area under the curve of 0.889 and 0.915 for NCEP/ATP and IDF, respectively). LAP performed the closest agreement in discriminating MetS-NCEP/ATP $(K=0.598, p-v a l u e<0.001)$ and MetS-IDF $(\kappa=0.577, p-v a l u e<0.001)$. Logistic regression analysis exhibited a strong association of the LAP cut-off point with MetS, with the odds ratio being from 23.37 to 27.22 (p-value<0.001).

Contact: Wantanee Kriengsinyos, R.D., Ph.D. Institute of Nutrition, Mahidol University, Phutthamonthon, Nakhon Pathom 73170, Thailand.

E-mail: wantanee.krieng@mahidol.ac.th

This is an open access article under the CC BY-NC-ND license

(http://www.jhsmr.org/index.php/jhsmr/about/editorialPolicies\#openAccessPolicy).

J Health Sci Med Res 2021;39(4):331-343 doi: $10.31584 / j h s m r .2021791$ www.jhsmr.org 
Conclusion: These study results revealed that LAP was strongly associated with MetS, had an outstanding and reliable diagnostic accuracy for discriminating MetS in Thai adults, which might be helpful for early detection of MetS among vulnerable populations.

Keywords: adiposity index, cut-off point, metabolic syndrome, lipid accumulation product, Thai adults

\section{Introduction}

Metabolic syndrome (MetS) had contributed to the major causes of cardiovascular diseases, diabetes mellitus and even all-causes of mortality. ${ }^{1-3}$ Therefore, the diagnosis and treatment of the underlying risk factors for MetS appear to be a crucial strategy for the minimization of all-cause mortality associated with MetS in the general population. ${ }^{3}$ The prevalence of this disorder has continuously increased, and ranges between $24.0 \%$ to $32.6 \%$ by different definitions among Thai adults $\geq 35$ years. ${ }^{4}$ By tasking force on determining insulin resistance (IR), as a dominant cause of MetS, the World Health Organization (WHO) is an avantgarde to propose the MetS definition. ${ }^{5}$ Over time, several diagnostic criteria have been introduced, and have disclosed that MetS is an abnormality of co-occurrence of several risk factors, including abdominal obesity, hypertension, dyslipidemia, dysinsulinemia and IR. ${ }^{6}$ Currently, the diagnosis of MetS in the Thai population is based on The National Cholesterol Education Program/Adult Treatment Panel III (NCEP/ATP), or International Diabetes Federation (IDF). ${ }^{4}$ Consequently, individuals are diagnosed with MetS by undergoing five assessments of waist circumference (WC), blood pressure (BP), fasting blood glucose (FBG), serum triglyceride (TG) and high-density lipoprotein cholesterol (HDL-C). ${ }^{6}$ Therefore, it would be useful if there was a simpler, quicker and inexpensive indicator with high accuracy for early discriminating of MetS in the community.

Previous studies affirmed the strong association of body adiposity with obesity-related diseases. ${ }^{7}$ Lipid accumulation product (LAP), first introduced by Kahn, is a novel index based on a combination of WC and TG. This index appeared to be an emergent indicator for assessing the central lipid accumulation. ${ }^{8}$ Both components (WC and TG) tend to increase with age; thus, their values subjectively accumulate over time: having more detrimental influences on the metabolic system. ${ }^{8}$ Numerous studies have been conducted to examine its diagnosis ability. LAP has been reported as being superior to other adiposity indicators, such as body mass index (BMI), WC, waist-to-hip ratio and waist-to-height ratio ( $\mathrm{WH} \mathrm{tR})$, to predict MetS among Spanish ${ }^{9}$, and Iranian populations. ${ }^{10}$ Results for the area under the curve (AUC) of 0.91 and 0.901 for LAP in discriminating MetS has been documented in healthy Argentinian ${ }^{11}$, and Taiwanese people $\geq 50$ years. ${ }^{12}$

Thai adults, have an increased risk of being diagnosed with MetS, due to their greater abdominal and visceral adiposity than Caucasians with a similar BMI. ${ }^{13}$ It has been suggested that central lipid distribution are distinct in different groups and ethnics; for instance, South Asians are more likely to accumulate additional fat in their abdomen and truncal part, which consequently possess greater WC, larger abdominal diameters, and thicker trunk skinfolds for a given weight in comparison with European individuals. ${ }^{14}$ Meanwhile, Asians and Japanese American subjects tend to deposit more intra-abdominal fat or visceral fat during weight gaining. ${ }^{14}$ Hence, early discrimination of MetS, with a simple and high accuracy index appears necessary. However, the application of the discriminating ability of LAP to MetS in the Thai population has not been investigated. 
By re-analyzing the National Health Examination Survey 2009 (NHES-IV), this present study's objectives were to determine the prevalence of MetS in Thai adults $\geq 18$ years and older. Furthermore, the study also assessed the MetS discriminatory ability of LAP, by employing numerous anthropometric and adiposity indices; including, traditional indices (BMI, WC, WHtR), non-traditional indices (cardiometabolic index (CMI) and the visceral adiposity index (VAI)).

\section{Material and Methods}

Study design and population: This present study analysed the database from NHES-IV. NHES-IV; ethical approval was performed by the Ethical Review Committee for Research in Human Subjects, Ministry of Public Health of Thailand. The design is described in the previous publication. ${ }^{15}$ Multistage probability cluster sampling technique was applied, and its sampling units were divided into four steps. Step one, 5 provinces in each of the 4 main regions (north, northeast, central, and south and Bangkok); step two, 2-3 districts in each selected province; step three, 13-14 electoral units (EU) or villages in each district; and step four, individuals among six age groups of each gender from each EU or village. A complete set of demographic and biochemistry variables were collected from all eligible subjects; with pregnancy being an exclusion factor. Finally, 18,642 Thai's 18 years: 8,910 males and 9,732 females, were analysed. Since the present study is a restrospective secondary data analysis conducted in 2018 , a request for exemption of informed consent for this study was submitted and approved by Institutional Review Board. The study protocol was approved by the Institutional Review Board of Mahidol University, Thailand (Protocol number MU-CIRB 2018/106.2105).

Data collection: A prior report described NHES-IV data collection procedures in detail. ${ }^{15}$ Key variables included demographic data; for example: age, education background, habitant's area; smoking and alcohol usage; medical history and physical activity patterns. Sitting BP was measured using an automatic monitor. Anthropometrical characteristics regarding body weight, height, WC, hip circumference $(\mathrm{HC})$ were collected. Body weight and height was measured using a calibrated digital scale and stadiometer, using standard procerdures. $\mathrm{HC}$ was measured at the height of the greater trochanter to the nearest $0.1 \mathrm{~cm}$ around the thighs, and WC was measured at a horizontal plane midway between the iliac crest and lower rib margin in centimeters to the nearest $0.1 \mathrm{~cm}$. Biochemical data were analyzed by fasting venous blood samples, which were collected in the morning from participants after 12-hours of fasting. TG was analyzed using the enzymatic colorimetric method; HDL-C, low-density lipoprotein cholesterol level (LDL-C) were analyzed by homogeneous enzymatic colorimetric methods; FBG were analyzed by the hexokinase enzyme method. All glucose and lipid parameters were carried out using the Hitachi 917 biochemistry analyzing machine (Roche Diagnostics, Basel, Switzerland).

Definitions: Individuals were diagnosed as having MetS when they meet the criteria by definitions of NCEP/ ATP and/or IDF. The NCEP/ATP defined that MetS individuals are a person who has $\geq 3$ metabolic abnormalities (BP $\geq 130 / 85 \mathrm{mmHg}, W C>90 / 80 \mathrm{~cm}$ for males/4emales, $\mathrm{TG} \geq 150 \mathrm{mg} / \mathrm{dL}, \mathrm{FPG} \geq 100 \mathrm{mg} / \mathrm{dL}, \mathrm{HDL}-\mathrm{C}<40 / 50 \mathrm{mg} / \mathrm{dL}$ for males/females. ${ }^{6}$ According to the IDF criteria, people are diagnosed with MetS when they have an essential component of central obesity; with WC $\geq 90 / 80 \mathrm{~cm}$ for males and females, respectively, together with having $\geq 2$ metabolic abnormalities $(B P \geq 130 / 85 \mathrm{mmHg}, T G \geq 150 \mathrm{mg} / \mathrm{dL}$, FPG $\geq 100 \mathrm{mg} / \mathrm{dL}, \mathrm{HDL}-\mathrm{C}<40 / 50 \mathrm{mg} / \mathrm{dL}$ for males/famales. ${ }^{6}$

Formulas: BMI was calculated by Quetelet's formula, as body weight $(\mathrm{kg})$ divided by height squared $\left(\mathrm{m}^{2}\right)$; WHtR was calculated by dividing WC $(\mathrm{cm})$ with height $(\mathrm{cm})$; LAP was calculated: [WC $(\mathrm{cm})-65] \times[T G(\mathrm{mM})]$ for males, [WC $(\mathrm{cm})-58] \times[\mathrm{TG}(\mathrm{mM})]$ for females; ${ }^{8}$ Cardiometabolic 
index (CMI) was calculated: TG/HDL-C $\times$ WHtR. ${ }^{16} \mathrm{VAl}$ was calculated as follows, males: VAI $=\{\mathrm{WC}(\mathrm{cm}) /[39.68+$ $(1.88 \times \mathrm{BMI})]\} \times(\mathrm{TG} / 1.03) \times(1.31 / \mathrm{HDL}-\mathrm{C})$; females: VAI $=\{\mathrm{WC}(\mathrm{cm}) /[39.58+(1.89 \times \mathrm{BMI})]\} \times(\mathrm{TG} / 0.81) \times(1.52 /$ HDL-C); wherein TG and HDL-C values were expressed in $\mathrm{mM}^{17}$

Statistical analysis: Complex survey analysis, including clustering, stratification and weighting, was employed to take into account the probability sampling design. Descriptive statistics were reported as ageadjusted arithmetic mean, with a 95\% confidence interval (95\% Cl). Demographical and biochemical parameters, between subjects having MetS and not having MetS, were compared using Mann-Whitney $U$ test or chi-squared test appropriately. Chi-squared test was used to examine the differences in MetS prevalence. The overall $p$ for a trend of MetS prevalence was analyzed by Mantel-Haenszel chi-square test. We used the cohen's kappa correlation coefficient to assess the agreement between WHtR, LAP and $\mathrm{CMI}$ and the references (NCEP/ATP and IDF). The interpretation suggested by Landis and Koch was used as follows: poor-to-fair agreement $(\kappa \leq 0.40)$, moderate agreement ( $\kappa$ of $0.41-0.60)$, substantial agreement ( $\kappa$ of 0.61-0.80) and excellent agreement ( $\kappa$ of $0.81-1.0$ ). Multiple logistic regression examined the association of WHtR, LAP and CMI, with the presence of MetS expressed by odds ratios (ORs), as categorized into per 1 standard deviation (S.D.) increment of the variable, and the cut-off point. Receiver-operating characteristic (ROC) curves evaluated the discriminating ability of adiposity indices to MetS. We determined an optimal cut-off value for ROC analysis to maximize Youden's index (J). The $\mathrm{J}$ can be defined as: maximum $=$ sensitivity + specificity $-1 .{ }^{18}$ The comparison of $\mathrm{AUC}$, for each indicator, were made via the recommendation of Hanley and McNeil. ${ }^{19}$ Values for each AUC could be ranged from 0 to 1, with a value of 1 indicating perfect diagnostic accuracy, and one with 0.5 having no discrimination power. Sensitivity (Sen), specificity (Spe), positive predictive value (PPV) and negative predictive value (NPV), for different indices' cut-off points, in discriminating MetS were calculated and expressed by percentages; with a 95\% Cl. IBM-SPSS Statistic for Windows, v 24.0 (IBM Corp., Armonk, New York, The United States of America) were applied for all statistical analyses: a $\mathrm{Cl}$ of $95 \%$ was adopted for all tests. Two-sided p-value $<0.050$ was considered statistically significant.

\section{Results}

Characteristics for the 18,642 subjects, with 8,910 males (47.8\%) and 9,732 females (52.2\%), are presented in Table 1. Regardless of gender, Thai adults having MetS were significantly older; lived abundantly in urban areas; appeared to be lower educated and conduced less physical activities; had higher body weights, HC and BP. Despite having lower smoking and alcohol consumption, they appeared to have less favorable glucose and lipid metabolism profiles (high FBG, high TG, high LDL-C in addition to low HDL-C) than people without the presence of MetS. Individuals having MetS showed increases in all adiposity indicators: specifically BMI, WC, WHtR, LAP, CMI and $\mathrm{VAI}$, in comparison to those not having MetS.

The age-adjusted prevalence of MetS among Thai adults, by different definitions, is reported in Table 2. Overall, $20.0 \%$ to $27.0 \%$ of adults were diagnosed with MetS; depending on each definition. The prevalence of NCEP/ ATP-defined MetS was 22.2\% (95\% Cl: 20.42-23.91) in males and $31.7 \%(95 \% \mathrm{Cl}: 29.89-33.42)$ in females. A lower MetS prevalence was reported by IDF definition. IDF-defined MetS was given to $13.3 \%$ of males $(95 \% \mathrm{Cl}$ : $11.71-14.84)$ and $26.4 \%$ of females (95\% Cl: 24.62-28.21). An age-related increase in the presence of MetS was observed. The corresponding prevalence of MetS defined by NCEP/ATP and IDF among adults aged 18-29 was 9.6\% (95\% Cl: 7.93-11.25) and 8.4\% (95\% Cl: 6.70-10.08), 
Table 1 Characteristics of subjects having metabolic syndrome and not having metabolic syndrome, according to the National Cholesterol Education Program/Adult Treatment Panel-III definition

\begin{tabular}{|c|c|c|c|}
\hline Variables & Not having MetS & Having MetS & p-value \\
\hline Number of subjects (person) & 12,641 & 6,001 & - \\
\hline Age (years) & $43.2(42.7-43.7)$ & $51.3(50.8-51.9)$ & $<0.001$ \\
\hline \multicolumn{4}{|l|}{ Living area } \\
\hline Rural & $70.6(62.9-77.3)$ & $67.0(59.0-74.2)$ & $<0.001$ \\
\hline Urban & $29.4(22.7-37.1)$ & $33.0(25.8-41.0)$ & $<0.001$ \\
\hline \multicolumn{4}{|l|}{ Education background } \\
\hline Illiterate & $3.7(3.1-4.4)$ & $4.5(3.9-5.3)$ & $<0.001$ \\
\hline Primary school & $58.0(55.3-60.7)$ & $71.3(69.0-73.5)$ & $<0.001$ \\
\hline Secondary and vocational & $31.4(29.6-33.2)$ & $19.8(18.0-21.7)$ & $<0.001$ \\
\hline University and higher & $6.9(5.9-8.1)$ & $4.4(3.5-5.5)$ & $<0.001$ \\
\hline Current regular smoking & $23.0(21.5-24.5)$ & $15.2(14.2-16.3)$ & $<0.001$ \\
\hline Alcohol drinking consumption within 12 months & $48.8(46.6-50.9)$ & $37.4(35.5-39.5)$ & $<0.001$ \\
\hline \multicolumn{4}{|l|}{ Alcohol drinking consumption level } \\
\hline Abstainer & $63.8(62.1-65.4)$ & $74.2(72.4-76.0)$ & $<0.001$ \\
\hline Low risk & $27.1(25.2-29.0)$ & $17.3(15.7-19.0)$ & $<0.001$ \\
\hline Moderate risk & $2.9(2.5-3.4)$ & $2.5(1.9-3.3)$ & $<0.001$ \\
\hline High risk & $2.1(1.8-2.4)$ & $1.9(1.5-2.6)$ & $<0.001$ \\
\hline Severe risk & $2.6(2.2-3.0)$ & $1.8(1.4-2.4)$ & $<0.001$ \\
\hline Drinking N/A amount & $1.5(1.1-2.1)$ & $2.2(1.5-3.3)$ & $<0.001$ \\
\hline \multicolumn{4}{|l|}{ Physical activity level } \\
\hline Low level & $17.5(15.5-19.8)$ & $20.3(18.2-22.6)$ & $<0.001$ \\
\hline Moderate level & $24.7(23.5-26.1)$ & $27.1(25.8-28.6)$ & $<0.001$ \\
\hline High level & $57.8(55.1-60.3)$ & $52.6(50.1-55.0)$ & $<0.001$ \\
\hline Height (cm) & $159.3(159.0-159.5)$ & $158.1(157.7-158.4)$ & $<0.001$ \\
\hline Body Weight (kg) & $57.8(57.3-58.2)$ & $68.4(67.6-69.2)$ & $<0.001$ \\
\hline $\operatorname{BMI}\left(\mathrm{kg} / \mathrm{m}^{2}\right)$ & $22.7(22.6-22.9)$ & $27.3(27.0-27.5)$ & $<0.001$ \\
\hline WC (cm) & $76.4(75.9-76.9)$ & $89.3(88.7-90.0)$ & $<0.001$ \\
\hline $\mathrm{HC}(\mathrm{cm})$ & $91.0(90.6-91.3)$ & $98.9(98.5-99.4)$ & $<0.001$ \\
\hline $\mathrm{SBP}(\mathrm{mmHg})$ & $118.4(117.8-119.1)$ & $134.2(133.1-135.3)$ & $<0.001$ \\
\hline $\mathrm{DBP}(\mathrm{mmHg})$ & $73.6(73.1-74.0)$ & $81.7(81.2-82.3)$ & $<0.001$ \\
\hline $\mathrm{FBG}(\mathrm{mg} / \mathrm{dL})$ & $85.6(84.2-87.0)$ & $105.3(102.7-107.8)$ & $<0.001$ \\
\hline $\mathrm{TG}(\mathrm{mg} / \mathrm{dL})$ & $125.8(120.8-130.8)$ & $231.2(222.6-239.7)$ & $<0.001$ \\
\hline $\mathrm{HDL}-\mathrm{C}(\mathrm{mg} / \mathrm{dL})$ & $49.2(48.4-50.1)$ & $40.7(40.1-41.3)$ & $<0.001$ \\
\hline LDL-C (mg/dL) & $126.7(124.5-128.9)$ & $133.6(130.5-136.7)$ & $<0.001$ \\
\hline WHtR & $0.48(0.47-0.48)$ & $0.57(0.56-0.57)$ & $<0.001$ \\
\hline LAP & $21.5(20.7-22.3)$ & $73.4(70.3-76.6)$ & $<0.001$ \\
\hline $\mathrm{CMI}$ & $1.37(1.29-1.45)$ & $3.47(3.30-3.63)$ & $<0.001$ \\
\hline VAl & $1.76(1.67-1.85)$ & $4.25(4.03-4.48)$ & $<0.001$ \\
\hline
\end{tabular}

Value is expressed as mean (interquartile range) or percentage $(95 \% \mathrm{Cl})$, as appropriate. The p-value denotes significant differences between subjects having MetS and not having MetS. Differences were compared by Mann-Whitney $U$ test, or chi-square test appropriately.

BMI=Body Mass Index, CMI=Cardiometabolic Index, DBP=Diastolic Blood Pressure, FBG=Fasting Blood Glucose, HC=Hip Circumference, HDL-C=High density lipoprotein cholesterol, MetS=Metabolic Syndrome, NCEP/ATP III=National Cholesterol Education Program/Adult Treatment Panel-III, LAP=Lipid Accumulation Product, LDL-C=Low density lipoprotein cholesterol, SBP=Systolic Blood Pressure, TG=Triglyceride, VAl=Visceral Adiposity Index, WC=Waist Circumference, WHtR=Waist-to-Height Ratio 
Table 2 Age-adjusted prevalence of metabolic syndrome among Thai adults, by the National Cholesterol Education Program/Adult Treatment Panel-III and International Diabetes Federation definition

\begin{tabular}{|c|c|c|c|c|c|}
\hline \multirow{2}{*}{ Definition } & \multirow{2}{*}{ Overall subjects } & \multicolumn{3}{|c|}{ Age (years) } & \multirow{2}{*}{$p$ for trend } \\
\hline & & $18-29$ & $30-59$ & $\geq 60$ & \\
\hline \multicolumn{6}{|l|}{ Both genders } \\
\hline NCEP/ATP & $27.0(25.6-28.5)$ & $9.6(7.9-11.3)$ & $27.6(26.0-29.1)$ & $41.3(39.7-42.9)$ & $<0.001$ \\
\hline IDF & $20.0(18.6-21.5)$ & $8.4(6.7-10.1)$ & $21.0(19.5-22.4)$ & $26.9(25.1-28.7)$ & $<0.001$ \\
\hline \multicolumn{6}{|l|}{ Males } \\
\hline NCEP/ATP & $22.2(20.4-23.9)$ & $9.2(7.5-11.0)$ & $23.5(21.5-25.6)$ & $30.3(28.5-32.2)$ & $<0.001$ \\
\hline IDF & $13.3(11.7-14.8)$ & $7.2(5.3-9.0)$ & $14.2(12.6-15.9)$ & $15.7(13.3-18.0)$ & $<0.001$ \\
\hline \multicolumn{6}{|l|}{ Females } \\
\hline NCEP/ATP & $31.7(29.9-33.4)$ & $10.0(7.6-12.5)$ & $31.3(29.4-33.3)$ & $50.3(48.5-52.2)$ & $<0.001$ \\
\hline IDF & $26.4(24.6-28.2)$ & $9.8(7.4-12.3)$ & $27.3(25.4-29.2)$ & $36.1(34.0-38.2)$ & $<0.001$ \\
\hline
\end{tabular}

Values are expressed as prevalence, with a $95 \% \mathrm{Cl}$ indicated in the parentheses. The chi-square test compared the prevalence between males and females corresponding MetS definition and among different age-groups (all $p$-value $<0.001$ ). The overall $p$ for trend of MetS prevalence was analyzed by Mantel-Haenszel chi-square test.

IDF=International Diabetes Federation, NCEP/ATP=The National Cholesterol Education Program/Adult Treatment Panel III

respectively; for those aged $30-59$, it was $27.6 \%$ (95\% Cl: 26.04-29.06) and 21.0\% (95\% Cl: 19.53-22.38); and for people $\geq 60$ years, it was $41.3 \%$ (95\% Cl: 39.73-42.93) and $26.9 \%$ (95\% Cl: 25.08-28.73)

Table 3 illustrates the discriminating ability of different adiposity indicators for MetS, by different definitions. The top four excellent abilities to discriminate MetS-NCEP/ATP in Thai adults aged $\geq 18$ years old and older was for LAP (AUC: 0.889; 95\% Cl: 0.884-0.894), followed by CMI (AUC: $0.871 ; 95 \% \mathrm{Cl}: 0.866-0.876)$. The third best performance was VAI (AUC: 0.868; 95\% Cl: 0.863-0.873), followed by WHtR (AUC: 0.823; 95\% Cl: 0.817-0.830). By applying IDF, WHtR appeared to be the most reliable indicators in discriminating MetS (AUC: 0.926; 95\% Cl: 0.922-0.929), the second-best discriminating ability was for LAP (AUC: 0.915; 95\% Cl: 0.911-0.919). Notably, the AUC value of CMI and VAI was decreased, which was significantly inferior to BMI ( $p$-value $<0.001)$. The influence of age on the discriminating ability was explored in stratified analyses (ages 18-29, 30-59, and $\geq 60$ years), and the results exhibited that the overall trend did not vary substantially by age (data was omitted). Regarding the optimal cut-off point, the best value for LAP cut-off point in this study population to discriminate MetS according to NCEP/ATP and IDF criteria was 34.38 $\mathrm{cm} . \mathrm{mmol} / \mathrm{L}$ and $37.96 \mathrm{~cm} . \mathrm{mmol} /$, respectively.

As shown in Table 3, the indicators with highest AUC value were LAP, CMI, VAI, and WHtR. These indices were selected for further exploration. However, VAI was not chosen due to its complication (with five components), expensiveness, and being statistically inferior to LAP in discriminating MetS among Thai adults. Therefore, WHtR, LAP and CMI were further analyzed. Cohen's Kappa coefficients between WHtR, LAP and CMI, and two references (NCEP/ATP and IDF) in the discrimination of MetS in Thai adults aged $\geq 18$ years old and older were performed (Table 4). Overall, LAP performed with moderate agreements in both references $(\kappa$-values were 0.577 and 0.598, with IDF and NCEP/ATP, respectively); whereas, $\mathrm{CMI}$ and $\mathrm{WH} \mathrm{HR}$ showed good agreement with only one definition. Specifically, the highest agreement values for MetS-NCEP/ATP were reported in LAP $(K=0.598$, p-value $<0.001)$, followed by $\mathrm{CMI}(\kappa=0.567, \mathrm{p}-$ value $<0.001)$, and 


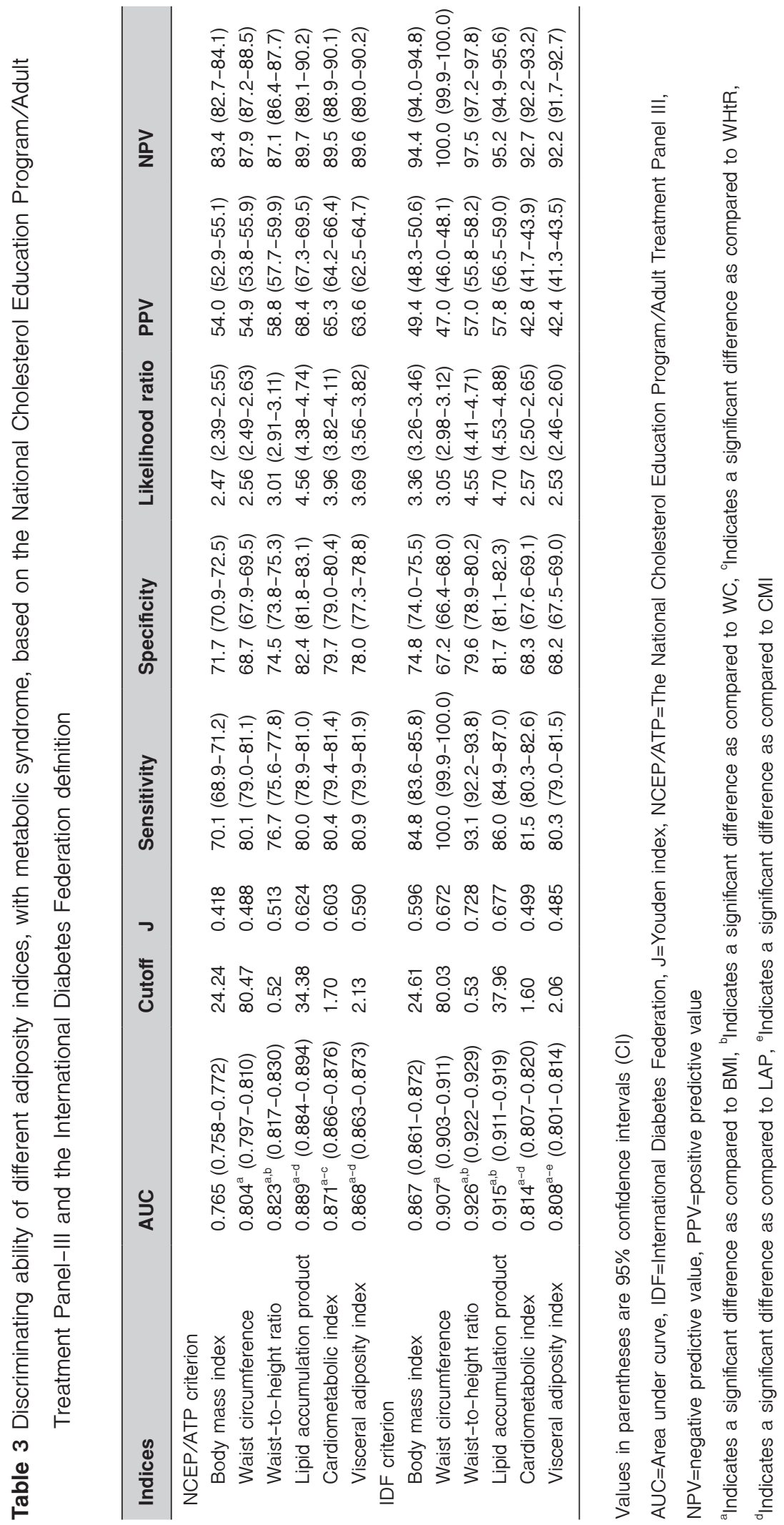


the lowest of $\mathrm{WHtR}(\kappa=0.474, \mathrm{p}$-value $<0.001)$. Whereas, the coefficient values for MetS-IDF were WHtR $(\kappa=0.593$, p-value $<0.001)$, followed by LAP $(\kappa=0.577$, p-value $<0.001$ ), and poor-to-fair agreement was observed in CMI $(\kappa=0.378, p-$ value $<0.001)$.

Table 4 Cohen's kappa coefficient $(\kappa)$ between standard definitions (NCEP/ATP or IDF) and adiposity indices (WHtR, LAP, and CMI)

\begin{tabular}{lllll}
\hline & $\begin{array}{l}\text { Cohen's } \\
\text { kappa } \\
\text { Variables }\end{array}$ & SE & p-value \\
& $(\mathbf{K})$ & & \\
\hline NCEP/ATP definition & & & \\
WHtR (Cut-off point >0.52) & 0.474 & 0.006 & $<0.001$ \\
LAP (Cut-off point >34.38) & 0.598 & 0.006 & $<0.001$ \\
CMI (Cut-off point >1.70) & 0.567 & 0.006 & $<0.001$ \\
IDF definition & & & \\
WHtR (Cut-off point >0.53) & 0.593 & 0.006 & $<0.001$ \\
LAP (Cut-off point >37.96) & 0.577 & 0.006 & $<0.001$ \\
CMI (Cut-off point >1.60) & 0.378 & 0.006 & $<0.001$ \\
\hline
\end{tabular}

Values are expressed as Cohen's Kappa coefficient value $(\kappa)$. $\mathrm{CMI}=$ Cardiometabolic Index, IDF=International Diabetes Federation (IDF), NCEP/ATP=The National Cholesterol Education Program/Adult Treatment Panel III (NCEP/ATP), LAP=Lipid Accumulation Product, $\mathrm{SE}=$ Standard error, WHtR=Waist-to-Height Ratio

Table 5 exhibits the multiple adjusted association between WHtR, LAP and CMI, and the prevalence of MetS. The result revealed that LAP consistently remained as having a robust association with MetS in all models. For each, additional 1 S.D. increment of the adiposity index score in the simplest model (model 1), the OR of MetSNCEP/ATP were raised from 4.84 to 21.10; as highest from LAP, OR: 21.10 (95\% Cl: 17.90-24.87), followed by CMI, OR: 6.17 (95\% Cl: 5.57-6.83); and WHtR, OR: 4.84 (95\% Cl: 4.41-5.32). By applying the IDF definition, LAP continuously exhibited the highest association with MetS, by OR of 32.00 (95\% Cl: $28.00-36.56)$ value, followed by
WHtR (OR: 11.24) and CMI (OR: 3.68). After adjusting for age, demographic, lifestyle and biochemical confounding factors, we observed the increment of OR is associated with MetS in LAP and CMl; whereas, reduction in WHtR [LAP, OR: 22.09 (95\% Cl: 18.18-26.85); CMI, OR: 8.56 (95\% Cl: 7.76-9.45) and WHtR, OR: 4.70 (95\% Cl: 4.23-5.23); all p-values $<0.001]$. Similar trending was observed in the IDF definition.

These results revealed that LAP was strongly associated with the odds of the presence of MetS by both definitions. The association between abnormal adiposity indices (defined by excess of the optimal cut-off value) and MetS was also analyzed. In the most complex model (model 3), Thai adults having abnormal adiposity indicators were approximately 10-23 times and 11-42 times more likely to have MetS (defined by NCEP/ATP and IDF, respectively) than those at the normal cut-off point. Specifically, the highest $\mathrm{OR}$ indicating the strongest association with MetS-NCEP/ATP were for CMI and LAP [OR: 23.39 (95\% Cl: 20.81-26.30) and OR: 23.37 (95\% Cl: 20.61-26.49), respectively]; whereas, WHtR had a lower association (OR: $10.3895 \% \mathrm{Cl}$ : 9.23-11.67). Inversely, the cut-off point of WHtR performed as having the strongest association with MetS-IDF (OR: 42.51), followed by LAP (OR: 27.22) and CMI (OR: 4.40).

\section{Discussion}

Our findings revealed that LAP has outstanding discriminating ability to MetS, with an AUC of 0.889 and 0.915, by NCEP/ATP and IDF definitions. Compared to other advanced adiposity indicators (for instance. VAI, and CMI), LAP appeared to be a simpler indicator, requiring only two components of TG and WC. This present study indicated the nationwide age-adjusted prevalence of MetS-NCEP/ATP and MetS-IDF among Thai adults as $27.0 \%$ and $20 \%$, respectively. These prevalence are lower than previous studies in Thailand as well as in other Asian 
Table 5 Multivariate logistic regression of WHtR, LAP and CMl for the presence of metabolic syndrome, defined by the National Cholesterol Education Program/Adult Treatment Panel-III and International Diabetes Federation definition in Thai adults aged $\geq 18$ years old and older

\begin{tabular}{|c|c|c|c|c|c|c|}
\hline Variable & $\begin{array}{l}\text { Model } 1 \\
\text { Odds ratio }(95 \% \mathrm{Cl})\end{array}$ & $p$-value & $\begin{array}{l}\text { Model } 2 \\
\text { Odds ratio }(95 \% \mathrm{Cl})\end{array}$ & p-value & $\begin{array}{l}\text { Model } 3 \\
\text { Odds ratio }(95 \% \mathrm{Cl})\end{array}$ & p-value \\
\hline \multicolumn{7}{|l|}{ NCEP/ATP definition } \\
\hline \multicolumn{7}{|l|}{ WHtR } \\
\hline Cut-off point $>0.52$ & $11.10(10.00-12.31)$ & $<0.001$ & $11.28(10.19-12.49)$ & $<0.001$ & $10.38(9.23-11.67)$ & $<0.001$ \\
\hline Per 1 S.D. increment & $4.84(4.41-5.32)$ & $<0.001$ & $5.04(4.59-5.52)$ & $<0.001$ & $4.70(4.23-5.23)$ & $<0.001$ \\
\hline \multicolumn{7}{|l|}{ LAP } \\
\hline Cut-off point $>34.38$ & $23.03(20.53-25.84)$ & $<0.001$ & 23.21 (20.66-26.07) & $<0.001$ & $23.37(20.61-26.49)^{*}$ & $<0.001$ \\
\hline Per 1 S.D. increment ${ }^{a}$ & $21.10(17.90-24.87)$ & $<0.001$ & $22.28(18.78-26.44)$ & $<0.001$ & $22.09(18.18-26.85)^{*}$ & $<0.001$ \\
\hline \multicolumn{7}{|l|}{ CMI } \\
\hline Cut-off point $>1.70$ & $16.21(14.60-18.00)$ & $<0.001$ & $18.62(16.50-21.03)$ & $<0.001$ & 23.39 (20.81-26.30) & $<0.001$ \\
\hline Per 1 S.D. increment ${ }^{a}$ & 6.17 (5.57-6.83) & $<0.001$ & $7.23(6.55-7.97)$ & $<0.001$ & $8.56(7.76-9.45)$ & $<0.001$ \\
\hline \multicolumn{7}{|l|}{ IDF definition } \\
\hline \multicolumn{7}{|l|}{$\mathrm{WHtR}$} \\
\hline Cut-off point $>0.53$ & $48.01(40.78-56.53)$ & $<0.001$ & 46.38 (39.47-54.49) & $<0.001$ & $42.51(35.83-50.43)$ & $<0.001$ \\
\hline Per 1 S.D. increment & $11.24(9.48-11.33)$ & $<0.001$ & $11.34(9.51-13.52)$ & $<0.001$ & 10.87 (9.02-13.09) & $<0.001$ \\
\hline \multicolumn{7}{|l|}{ LAP } \\
\hline Cut-off point $>37.96$ & 30.09 (26.53-34.14) & $<0.001$ & $31.82(28.15-35.97)$ & $<0.001$ & $27.22(24.07-30.77)$ & $<0.001$ \\
\hline Per 1 S.D. increment ${ }^{a}$ & 32.00 (28.00-36.56) & $<0.001$ & 41.39 (35.37-48.44) & $<0.001$ & 39.44 (33.36-46.63) & $<0.001$ \\
\hline \multicolumn{7}{|l|}{ CMI } \\
\hline Cut-off point $>1.60$ & 10.27 (9.12-11.57) & $<0.001$ & 12.04 (10.52-13.77) & $<0.001$ & $11.20(9.89-12.67)$ & $<0.001$ \\
\hline Per 1 S.D. increment ${ }^{a}$ & $3.68(3.42-3.97)$ & $<0.001$ & $4.35(4.02-4.70)$ & $<0.001$ & $4.40(4.09-4.74)$ & $<0.001$ \\
\hline
\end{tabular}

Model 1: adjusted for age

Model 2: further adjusted for living area, education background, current-regular smoking, current alcohol consumption, alcohol consumption level and physical activity.

Model 3: further adjusted for SBP, DBP, fasting blood glucose ${ }^{a}, L_{L}-C^{a}$.

'variables were log-transformed before analysis

Per S.D. increment: per 1 S.D. increment of log lipid accumulation product

Cut-off: The OR for presence of MetS in the comparison between above and below the cut-off value

$\mathrm{CMI}=$ Cardiometabolic Index, IDF=International Diabetes Federation, NCEP/ATP=The National Cholesterol Education Program/Adult Treatment Panel III, LAP=Lipid Accumulation Product, SE Standard error, WHtR=Waist-to-Height Ratio, SBP=Systolic blood pressure, DBP=diastolic blood pressure, LDL-C=low-density lipoprotein cholesterol

and Western countries. The InterASIA study reported that $32.6 \%$ and $24.0 \%$ of Thai's $\geq 35$ years old were diagnosed with MetS, defined by NCEP/ATP and IDF, respectively. ${ }^{4}$ Besides the prevalence of MetS, according to NCEP/ATP definition in 97,098 Chinese adults $\geq 18$ years was published at $33.9 \%{ }^{20}$, whereas, $31.3 \%$ of Korean ${ }^{21}$, and $46.1 \%$ of Srilankan adults had been diagnosed with MetS. ${ }^{22}$ On the other hand, a review analyzed among 34,821 subjects, from 10 European countries revealed that the prevalence of MetS in this continent was $24.3 \%$, by adopting the similar criteria of NCEP/ATP. ${ }^{23}$ Ethnicity, race and age considerably affects abdominal adiposity; especially large, central and visceral fat accumulation in Asians could account for the high prevalence of MetS among these populations. ${ }^{13,24}$ 
Our AUC results for LAP were consistent with previous findings, indicating the usefulness of LAP in discriminating MetS among different populations and ethnics. A study conducted among 552, healthy Argentinian men, reported the AUC for LAP in MetS-NCEP/ATP was $0.91^{11}$, LAP also exhibits the highest diagnostic accuracy for MetS-NCEP/ATP and MetS-IDF among Spanish and Iranian individuals. ${ }^{9,10}$ In Asian populations, LAP was revealed as an accurate predictor of MetS-NCEP/ATP in Taiwanese people $\geq 50$ years, with an AUC of $0.91 .^{12}$ The comparison of LAP with VAI and TyG also affirmed that LAP was a reliable indicator for MetS in Chinese people $\geq 40$ years, with an AUC of 0.855 and 0.865 ; defined by NCEP/ATP and IDF criteria, respectively. ${ }^{25}$ However, the AUC found in this study was slightly lower than those previously reported. The possible explanation for this might be the disparities in the ethnicity, race, surveyed population and sample size.

Notably, the ROC analysis in this study disclosed that $\mathrm{WHtR}$ was a potential index for discriminating MetS among Thai adults. WHtR showed a higher AUC value than LAP in discriminating MetS-IDF; however, it was inferior in discriminating MetS-NCEP/ATP. Analyses on Cohen's kappa coefficient agreement showed that WHtR might be applied to discriminate MetS-IDF only $(K=0.593)$, but not for MetS-CEP/ATP ( $K=0.474)$; whereas, LAP performed with moderate agreement in both definitions $(K=0.577$ and 0.598 in IDF and NCEP/ATP, respectively. Multiple regression analysis indicated a similar observation. The OR between the cut-off point of WHtR and MetS presence fluctuated (OR of 10.38 and 42.51 in MetS-NCEP/ATP and IDF, respectively, while in LAP these ratios were 23.37 and 27.22).

Although, WHtR required only two, unmixed anthropometric components of WC and height, the use of LAP as a discriminating tool for MetS appeared more advantageous. First, by assessing total body fat, WHtR is unable to classify between subcutaneous adipose tissue (SAT) and intraabdominal fat or visceral adipose tissue (VAT). ${ }^{26}$ Comparing with SAT, VAT is more strongly associated, has more detrimental influences with cardio-metabolic risks and is firmly corroborated for MetS prediction. ${ }^{27,28}$ The accumulation of excessive VAT adipocytes may result in possessing higher rates of lipolysis, producing extra adipocytokines; for instance, tumor necrosis factor-alpha (TNF-a), interleukin-6 (IL-6), IL-1, and plasminogen activator inhibitor-1 (PAI-1) and so forth ${ }^{29}$, which are more prone to develop insulin resistance, as well as to escalate inflammation, than SAT adipocytes. ${ }^{30}$ Second, the measurement of WHtR brings confrontations in both height and WC components. In adult populations, height is a constant variable; thus, the assessment of WHtR was considerably affected by the WC measurement. Any potential under- or over-evaluation of WC may make a sizable miscalculation. Indeed, the measurement of WC and its calibration still faces challenges (examples; assistance requirements, time-consumption, measuring in obese or bed-ridden patients); ${ }^{31}$ whereas, the height variable revealed its limitation in assessing people with anatomy and physical disabilities (examples; frail individuals, subjects with amputation, wheelchair users and so on). ${ }^{26}$ Furthermore, several criticisms dismiss $\mathrm{WHtR}$ in its application for monitoring, such as its vague biological interpretation, its greater variability across age; especially in elderly people, gender, race and ethnic groups, additionally its fluctuation waist values depend on weight change. ${ }^{26}$

LAP, comprised of $W C$ and $T G$, showed more potential in assessment; markedly in the role of $\mathrm{TG}^{8}$ Inflammation and insulin resistance played a crucial role in the pathogenic mechanisms of MetS. ${ }^{6}$ TG, accurate measurement by biochemical testing, has been reported to be a robust, positive determinant associated with VAT in both healthy and obese adults: even after controlling for SAT. ${ }^{32,33}$ Distinctly, previous studies indicated that the turnover of TG in VAT is a reliable indicator of metabolic 
risk, that was not related to the adipokine release, or to be taken into consideration as to the size of SAT, as well as the pathogenic characteristics of this tissue..$^{30}$ Moreover, the relationship between TG and insulin is well-characterized. ${ }^{34}$ Insulin facilitates the stimulation of glucose and fatty acid uptake, which intensifies the TG synthesis and esterification processes. ${ }^{34}$ Therefore, LAP proposed to estimate visceral fat accumulation, and therefore the significantly higher levels of LAP may mediate less favorable IR, cardiovascular risks and metabolic profiles. ${ }^{8}$ Furthermore, the combination of TG with WC; termed: triglyceridemic-waist, has been applied to discriminating individuals with the excess of $\mathrm{VAT}^{35}$, and is associated with an increased risk of MetS. ${ }^{36}$ However, visceral accumulation may not be described adequately by a dichotomous index, as obesity itself is a continuous process. ${ }^{8}$ Indeed, it has been suggested that accurate detection of discrete metabolic conditions requires models, composed by continuous rather than dichotomous risk factors. LAP appeared to be more advantageous by formulating to be a remarkable, continuous index, to reflect the combined anatomic and physiological changes associated with visceral fat deposition. ${ }^{8}$

There are some limitations in this study. First, we were unable to assign causality to our findings, due to the nature of the cross-sectional study. Second, given that our study was principally conducted among Thai adults, it is uncertain to extrapolating these findings to other racial or ethnic populations. Nevertheless, several strengths in the study should be acknowledged. Firstly, the study is a national survey, with a large, representative sample. Secondly, we used standardized protocols and data collection procedures, the data collectors were all trained and there was quality control assurance. Additionally, all blood samples were analyzed in a central laboratory in Bangkok, according to clinic laboratory standards. Hence, all of the abovementioned factors largely avoided measurement bias.

\section{Conclusion}

This is the first study to investigate the discriminating performance of adiposity indices to MetS in Thai adults. LAP was strongly associated and found to be an accurate index for discriminating MetS. Our findings suggest that this simple marker may help, in a primary care setting, to identify subjects who require further biochemical evaluation in MetS examinations.

\section{Acknowledgement}

The NHES IV was conducted by the NHES Office, Health Systems Research Institute, Thailand. NHES IV was supported by the Health System Research Institute; the Bureau of Policy and Strategy, Ministry of Public Health; the Thai Health Promotion Foundation; and the National Health Security Office, Thailand.

\section{Funding sources}

This present study received no funding.

\section{Conflict of interest}

All authors have no conflicts of interest to declare for this study.

\section{References}

1. Mottillo S, Filion KB, Genest J, Joseph L, Pilote L, Poirier P, et al. The metabolic syndrome and cardiovascular risk a systematic review and meta-analysis. J Am Coll Cardiol 2010;56:1113-32.

2. Ford ES, Li C, Sattar N. Metabolic syndrome and incident diabetes: current state of the evidence. Diabetes Care 2008; 31:1898-904.

3. Wu SH, Liu Z, Ho SC. Metabolic syndrome and all-cause mortality: a meta-analysis of prospective cohort studies. Eur J Epidemiol 2010;25:375-84.

4. Aekplakorn W, Chongsuvivatwong V, Tatsanavivat P, Suriyawongpaisal P. Prevalence of metabolic syndrome defined by the International Diabetes Federation and National Cholesterol Education Program criteria among Thai adults. Asia Pac J Public Health 2011;23:792-800. 
5. Alberti KG, Zimmet PZ. Definition, diagnosis and classification of diabetes mellitus and its complications. Part 1: diagnosis and classification of diabetes mellitus provisional report of a WHO consultation. Diabet Med 1998;15:539-53.

6. Alberti KG, Eckel RH, Grundy SM, Zimmet PZ, Cleeman JI, Donato KA, et al. Harmonizing the metabolic syndrome: a joint interim statement of the International Diabetes Federation Task Force on Epidemiology and Prevention; National Heart, Lung, and Blood Institute; American Heart Association; World Heart Federation; International Atherosclerosis Society; and International Association for the Study of Obesity. Circulation 2009;120:1640-5.

7. Jung UJ, Choi MS. Obesity and its metabolic complications: the role of adipokines and the relationship between obesity, inflammation, insulin resistance, dyslipidemia and nonalcoholic fatty liver disease. Int J Mol Sci 2014;15:6184-223.

8. Kahn HS. The "lipid accumulation product" performs better than the body mass index for recognizing cardiovascular risk: a population-based comparison. BMC Cardiovasc Disord. 2005;5:26.

9. Taverna MJ, Martinez-Larrad MT, Frechtel GD, Serrano-Rios M. Lipid accumulation product: a powerful marker of metabolic syndrome in healthy population. Eur J Endocrinol 2011; 164:559-67.

10. Motamed N, Razmjou S, Hemmasi G, Maadi M, Zamani F. Lipid accumulation product and metabolic syndrome: a populationbased study in northern Iran, Amol. J Endocrinol Invest 2016; 39:375-82.

11. Tellechea ML, Aranguren F, Martinez-Larrad MT, Serrano-Rios M, Taverna MJ, Frechtel GD. Ability of lipid accumulation product to identify metabolic syndrome in healthy men from Buenos Aires. Diabetes Care 2009;32:e85.

12. Chiang JK, Koo M. Lipid accumulation product: a simple and accurate index for predicting metabolic syndrome in Taiwanese people aged 50 and over. BMC Cardiovasc Disord 2012;12:78.

13. Lim U, Ernst T, Buchthal SD, Latch M, Albright CL, Wilkens LR, et al. Asian women have greater abdominal and visceral adiposity than Caucasian women with similar body mass index. Nutr Diabetes 2011;1:e6.

14. Pi-Sunyer FX. The epidemiology of central fat distribution in relation to disease. Nutr Rev 2004;62:S120-6.

15. Aekplakorn W, Chariyalertsak S, Kessomboon P, Sangthong R,
Inthawong R, Putwatana $\mathrm{P}$, et al. Prevalence and management of diabetes and metabolic risk factors in Thai adults: the Thai National Health Examination Survey IV, 2009. Diabetes Care 2011;34:1980-5.

16. Wakabayashi I, Daimon T. The "cardiometabolic index" as a new marker determined by adiposity and blood lipids for discrimination of diabetes mellitus. Clin Chim Acta 2015;438: 274-8.

17. Amato MC, Giordano C. Visceral adiposity index: an indicator of adipose tissue dysfunction. Int J Endocrinol 2014;2014: 730827.

18. Youden WJ. Index for rating diagnostic tests. Cancer 1950;3: 32-5.

19. Hanley JA, McNeil BJ. The meaning and use of the area under a receiver operating characteristic $(\mathrm{ROC})$ curve. Radiology 1982;143:29-36.

20. Lu J, Wang L, Li M, Xu Y, Jiang $Y$, Wang W, et al. Metabolic Syndrome Among Adults in China: The 2010 China Noncommunicable Disease Surveillance. J Clin Endocrinol Metab 2017;102:507-15.

21. Hong AR, Lim S. Clinical characteristics of metabolic syndrome in Korea, and its comparison with other Asian countries. J Diabetes Investig 2015;6:508-15.

22. Chackrewarthy S, Gunasekera D, Pathmeswaren A, Wijekoon $\mathrm{CN}$, Ranawaka UK, Kato N, et al. A Comparison between Revised NCEP ATP III and IDF Definitions in Diagnosing Metabolic Syndrome in an Urban Sri Lankan Population: The Ragama Health Study. ISRN Endocrinol 2013;2013:320176.

23. Scuteri A, Laurent S, Cucca F, Cockcroft J, Cunha PG, Mañas LR, et al. Metabolic syndrome across Europe: different clusters of risk factors. Eur J Prev Cardiol 2015;22:486-91.

24. Nazare JA, Smith JD, Borel AL, Haffner SM, Balkau B, Ross R, et al. Ethnic influences on the relations between abdominal subcutaneous and visceral adiposity, liver fat, and cardiometabolic risk profile: the International Study of Prediction of Intra-Abdominal Adiposity and Its Relationship With Cardiometabolic Risk/ntra-Abdominal Adiposity. Am J Clin Nutr 2012; 96:714-26.

25. Li R, Li Q, Cui M, Yin Z, Li L, Zhong T, et al. Clinical surrogate markers for predicting metabolic syndrome in middle-aged and elderly Chinese. J Diabetes Investig 2018;9:411-8.

26. Browning LM, Hsieh SD, Ashwell M. A systematic review of 
waist-to-height ratio as a screening tool for the prediction of cardiovascular disease and diabetes: 0.5 could be a suitable global boundary value. Nutr Res Rev 2010;23:247-69.

27. Hamdy O, Porramatikul S, Al-Ozairi E. Metabolic obesity: the paradox between visceral and subcutaneous fat. Curr Diabetes Rev 2006;2:367-73.

28. Kishida K, Funahashi T, Matsuzawa Y, Shimomura I. Visceral adiposity as a target for the management of the metabolic syndrome. Ann Med 2012;44:233-41.

29. Freedland ES. Role of a critical visceral adipose tissue threshold (CVATT) in metabolic syndrome: implications for controlling dietary carbohydrates: a review. Nutr Metab (Lond) 2004;1:12.

30. Carobbio S, Rodriguez-Cuenca S, Vidal-Puig A. Origins of metabolic complications in obesity: ectopic fat accumulation. The importance of the qualitative aspect of lipotoxicity. Curr Opin Clin Nutr Metab Care 2011;14:520-6.

31. Molarius A, Seidell JC. Selection of anthropometric indicators for classification of abdominal fatness--a critical review. Int J Obes Relat Metab Disord 1998;22:719-27.
32. Huang CY, Huang HL, Yang KC, Lee LT, Yang WS, Huang $\mathrm{KC}$, et al. Serum triglyceride levels independently contribute to the estimation of visceral fat amount among nondiabetic obese adults. Medicine (Baltimore) 2015;94:e965.

33. Nguyen-Duy TB, Nichaman MZ, Church TS, Blair SN, Ross R. Visceral fat and liver fat are independent predictors of metabolic risk factors in men. Am J Physiol Endocrinol Metab 2003; 284:E1065-71.

34. Czech MP, Tencerova M, Pedersen DJ, Aouadi M. Insulin signalling mechanisms for triacylglycerol storage. Diabetologia 2013;56:949-64.

35. Sam S, Haffner S, Davidson MH, D'Agostino RB Sr, Feinstein S, Kondos G, et al. Hypertriglyceridemic waist phenotype predicts increased visceral fat in subjects with type 2 diabetes. Diabetes Care 2009;32:1916-20.

36. Zainuddin LR, Isa N, Muda WM, Mohamed HJ. The prevalence of metabolic syndrome according to various definitions and hypertriglyceridemic-waist in malaysian adults. Int $\mathrm{J}$ Prev Med 2011;2:229-37. 